\title{
AP001056.1, A Prognosis-Related Enhancer RNA in Squamous Cell Carcinoma of the Head and Neck
}

\author{
Xiaolian Gu ${ }^{1, * \mathbb{D}}$, Lixiao Wang ${ }^{1}$, Linda Boldrup ${ }^{1}$, Philip J. Coates ${ }^{2}$, Robin Fahraeus ${ }^{1,2,3}$, \\ Nicola Sgaramella ${ }^{1}$, Torben Wilms ${ }^{4}$ and Karin Nylander ${ }^{1}(\mathbb{D}$ \\ 1 Department of Medical Biosciences/Pathology, Umeå University, 90185 Umeå, Sweden; \\ lixiao.wang@umu.se (L.W.); linda.boldrup@umu.se (L.B.); robin.fahraeus@inserm.fr (R.F.); \\ sgaramellanicola12@gmail.com (N.S.); karin.nylander@umu.se (K.N.) \\ 2 RECAMO, Masaryk Memorial Cancer Institute, 65653 Brno, Czech Republic; philip.coates@mou.cz \\ 3 Équipe Labellisée Ligue Contre le Cancer, INSERM UMRS1162, Institut de Génétique Moléculaire, \\ Université Paris 7, IUH Hôpital St. Louis, 75010 Paris, France \\ 4 Department of Clinical Sciences/ENT, Umeå University, 90185 Umeå, Sweden; Torben.Wilms@vll.se \\ * Correspondence: xiaolian.gu@umu.se
}

Received: 11 February 2019; Accepted: 7 March 2019; Published: 11 March 2019

\begin{abstract}
A growing number of long non-coding RNAs (lncRNAs) have been linked to squamous cell carcinoma of the head and neck (SCCHN). A subclass of lncRNAs, termed enhancer RNAs (eRNAs), are derived from enhancer regions and could contribute to enhancer function. In this study, we developed an integrated data analysis approach to identify key eRNAs in SCCHN. Tissue-specific enhancer-derived RNAs and their regulated genes previously predicted using the computational pipeline PreSTIGE, were considered as putative eRNA-target pairs. The interactive web servers, TANRIC (the Atlas of Noncoding RNAs in Cancer) and cBioPortal, were used to explore the RNA levels and clinical data from the Cancer Genome Atlas (TCGA) project. Requiring that key eRNAs should show significant associations with overall survival (Kaplan-Meier log-rank test, $p<0.05$ ) and the predicted target (correlation coefficient $r>0.4, p<0.001$ ), we identified five key eRNA candidates. The most significant survival-associated eRNA was AP001056.1 with ICOSLG encoding an immune checkpoint protein as its regulated target. Another 1640 genes also showed significant correlation with AP001056.1 ( $r>0.4, p<0.001)$, with the "immune system process" being the most significantly enriched biological process (adjusted $p<0.001$ ). Our results suggest that AP001056.1 is a key immune-related eRNA in SCCHN with a positive impact on clinical outcome.
\end{abstract}

Keywords: AP001056.1; lncRNA; enhancer; SCCHN; ICOSLG; tumor immunity

\section{Introduction}

Squamous cell carcinoma of the head and neck (SCCHN) is the sixth most common cancer worldwide with an estimated 600,000 new cases and 300,000 deaths annually [1]. SCCHN comprises a group of cancers located in the oral cavity, nasal cavity, larynx, hypopharynx and oropharynx. Tobacco and alcohol consumption, and human papillomavirus (HPV) infection are the most common predisposing factors for SCCHN [2,3].

Evidence is accumulating that long non-coding RNAs (lncRNAs) are linked to human diseases [4,5]. In contrast with the classic notion that non-protein-coding transcripts are non-functional and thus regarded as junk RNA, lncRNAs are biologically active and carry out diverse functions such as transcriptional regulation in cis or trans, the organization of nuclear domains and post-transcriptional regulation of proteins or RNA molecules [6,7]. Next-generation sequencing has identified tens of thousands of lncRNAs from single-cell eukaryotes to humans [8]. LncRNAs are expressed in a tissue 
and cell-type specific manner, making them potential cancer biomarkers [4] and many SCCHN-related lncRNAs have been reported based on differential expression at a given significance level $[9,10]$, even if the function of these disease-associated lncRNAs is largely unknown.

Enhancer RNAs (eRNA) are a subclass of lncRNAs transcribed within gene enhancers, a major type of cis-regulatory elements in the genome [11]. Potential models suggest that eRNAs interact with RNA polymerase II and transcription factors to facilitate promoter-enhancer looping and a consequent increase in the transcription of the corresponding downstream gene [12]. RNA synthesis from enhancers has been confirmed in the majority of human cells and tissues, and transcriptional regulation via eRNAs plays a role in cancer $[13,14]$. It has been shown in basal cell carcinoma that mutations in the eRNA elements of ACTRT1 could impair enhancer activity and ACTRT1 expression, leading to aberrant activation of Hedgehog signaling and the contribution to tumor development [15]. To the best of our knowledge, there are no reports on SCCHN-associated eRNAs [16].

Here we set out to identify prognostic eRNAs and their target genes in SCCHN. We found that the functionally unannotated long non-coding RNA AP001056.1, located within the tissue-specific enhancer of an immune checkpoint gene, ICOSLG, was significantly associated with survival in patients with SCCHN. High AP001056.1 levels in SCCHN associated with elevated expression of ICOSLG and other immune genes, suggesting that AP001056.1 is an immune-related eRNA with a positive impact on clinical outcome.

\section{Results}

\subsection{Putative Prognostic eRNAs in SCCHN}

Using the PreSTIGE algorithm, a total of 2695 ENCODE (Encyclopedia of DNA Elements database [17]) annotated lncRNA transcripts that are expressed from active tissue-specific enhancers, as well as 2303 predicted target genes, have previously been identified [18]. This transcript dataset was utilized for identifying putative eRNA-target pairs. To facilitate data exploration in TANRIC, a gene-based interactive web platform for exploring lncRNAs in cancer, we used Ensembl BioMart for conversion of transcript ID to gene ID. After that, the 2695 putative eRNA transcripts were mapped to their corresponding 1288 genes. Finally, according to RNA-sequencing data from 426 TCGA SCCHN patients provided by the TANRIC database, we identified 18 of the 1288 putative eRNA genes with levels significantly associated with overall survival (Table 1, Kaplan-Meier log-rank test, $p<0.05$ ). When correlating levels of these 18 eRNAs with levels of their predicted target gene mRNAs, significant correlations were seen only for five (Spearman's rank correlation coefficient $r>0.4, p<0.001$; Table 1 ).

\subsection{LncRNA AP001056.1 is a Key eRNA in SCCHN}

LncRNA AP001056.1 was found to be the top prognostic putative eRNA showing a positive correlation between gene expression and levels of its predicted target ICOSLG (inducible $\mathrm{T}$ cell costimulator ligand). In SCCHN patients, the AP001056.1-high group (cut off value not provided by TANRIC) showed better overall survival compared to the AP001056.1-low group (Figure 1a, Kaplan-Meier log-rank test, $p=0.002$ ). Additionally, AP001056.1 and ICOSLG mRNA levels correlated with each other (Figure 1b, Spearman's $r=0.504, p<0.001$ ). The prognostic effect of AP001056.1 and its correlation with ICOSLG mRNA levels in other TCGA cancer types was investigated using TANRIC. As summarized in Table 2, the impact of AP001056.1 on overall survival and ICOSLG was specific for SCCHN and glioblastoma only. 
Table 1. List of overall survival associated lncRNAs derived from enhancers.

\begin{tabular}{|c|c|c|c|c|c|}
\hline \multirow{2}{*}{ Ensembl ID } & \multirow{2}{*}{ Symbol } & \multirow{2}{*}{$\begin{array}{l}\text { TANRIC Overall } \\
\text { Survival Analysis, } \\
\text { Log-Rank } p \text {-Value }\end{array}$} & \multirow{2}{*}{ Predicted Target } & \multicolumn{2}{|c|}{$\begin{array}{l}\text { Correlation between lncRNA } \\
\text { and the Neighboring Target }\end{array}$} \\
\hline & & & & $p$-value & $\begin{array}{l}\text { Correlation } \\
\text { Coefficient } r\end{array}$ \\
\hline ENSG00000234902 & AC007879.3 & 0.000 & KLF7 & \multirow{4}{*}{\multicolumn{2}{|c|}{ No or weak correlation }} \\
\hline ENSG00000125514 & LINC00029 & 0.000 & SLC17A9 & & \\
\hline ENSG00000231419 & LINC00689 & 0.000 & WDR60 & & \\
\hline ENSG00000247982 & LINC00926 & 0.001 & CGNL1 & & \\
\hline ENSG00000237604 & AP001056.1 & 0.002 & ICOSLG & $<0.001$ & 0.504 \\
\hline ENSG00000259974 & LINC00261 & 0.002 & FOXA2 & \multirow{7}{*}{\multicolumn{2}{|c|}{ No or weak correlation }} \\
\hline ENSG00000250334 & LINC00989 & 0.003 & NAA11 & & \\
\hline ENSG00000229228 & LINC00582 & 0.007 & DISC1 & & \\
\hline ENSG00000245149 & RNF139-AS1 & 0.010 & NDUFB9 & & \\
\hline ENSG00000233208 & LINC00642 & 0.014 & CALM1 & & \\
\hline ENSG00000235126 & AC128709.2 & 0.016 & $B D H 1$ & & \\
\hline ENSG00000235192 & AC009495.3 & 0.019 & GALNT3 & & \\
\hline ENSG00000225783 & MIAT & 0.025 & CRYBB1 & $<0.001$ & 0.401 \\
\hline ENSG00000237819 & AC002454.1 & 0.027 & CDK6 & $<0.001$ & 0.671 \\
\hline ENSG00000179818 & PCBP1-AS1 & 0.030 & TIA1 & $<0.001$ & 0.537 \\
\hline ENSG00000236671 & PRKG1-AS1 & 0.037 & DKK1 & $<0.001$ & 0.713 \\
\hline ENSG00000244342 & LINC00698 & 0.039 & GALNT3 & \multirow{2}{*}{\multicolumn{2}{|c|}{ No or weak correlation }} \\
\hline ENSG00000227479 & AC124861.1 & 0.045 & GPC1 & & \\
\hline
\end{tabular}
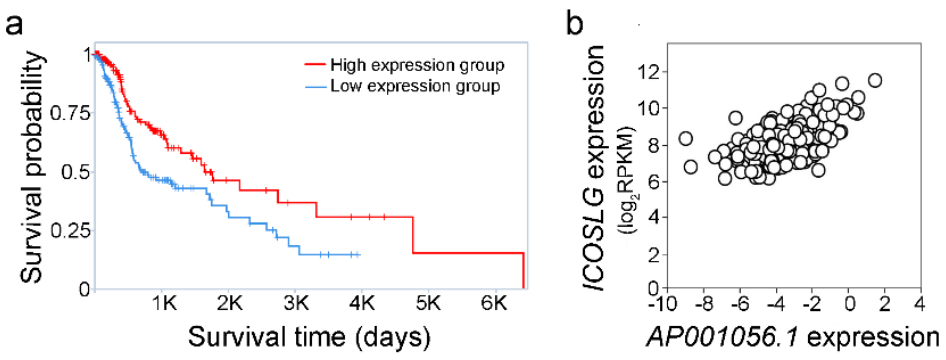

$\left(\log _{2}\right.$ RPKM)

Figure 1. Impact of lncRNA AP001056.1 on squamous cell carcinoma of the head and neck (SCCHN). (a) Kaplan-Meier overall survival curve for patients with AP001056.1-high and AP001056.1-low expression, obtained through the TANRIC (the Atlas of Noncoding RNAs in Cancer) platform. (b) Scatterplot showing the association between AP001056.1 and ICOSLG levels according to TANRIC correlation analysis.

\subsection{AP001056.1 Expression is Subsite Specific and Associates with HPV Status}

We further investigated AP001056.1 transcript levels in different SCCHN subsites and found significant differences (Figure 2a, Kruskal-Wallis H analysis, $p=0.014$ ), with the highest median level seen in tonsillar cancer ( 31 patients). 
Table 2. TANRIC survival analysis and gene expression correlations for AP001056.1 and ICOSLG across 20 cancer types from the Cancer Genome Atlas (TCGA) project.

\begin{tabular}{|c|c|c|c|c|}
\hline \multicolumn{2}{|r|}{ Tumor Type } & \multirow{2}{*}{$\begin{array}{c}\text { AP001056.1 and } \\
\text { Overall Survival } \\
\text { Log-Rank } p \text {-Value }\end{array}$} & \multicolumn{2}{|c|}{ AP001056.1 and ICOSLG } \\
\hline Abbreviation & Detail & & $\begin{array}{c}\text { Correlation } \\
p \text {-Value }\end{array}$ & $\begin{array}{l}\text { Correlation } \\
\text { Coefficient }\end{array}$ \\
\hline HNSC & Head and Neck squamous cell carcinoma & 0.002 & $<0.001$ & 0.504 \\
\hline GBM & Glioblastoma multiforme & 0.035 & $<0.001$ & 0.571 \\
\hline KIRC & Kidney renal clear cell carcinoma & 0.094 & \multicolumn{2}{|c|}{ No or weak correlation } \\
\hline THCA & Thyroid carcinoma & 0.221 & \multicolumn{2}{|c|}{ No or weak correlation } \\
\hline LUSC & Lung squamous cell carcinoma & 0.254 & $<0.001$ & 0.494 \\
\hline LUAD & Lung adenocarcinoma & 0.262 & $<0.001$ & 0.510 \\
\hline BLCA & Bladder Urothelial Carcinoma & 0.345 & $<0.001$ & 0.559 \\
\hline OV & Ovarian serous cystadenocarcinoma & 0.352 & \multicolumn{2}{|c|}{ No or weak correlation } \\
\hline PRAD & Prostate adenocarcinoma & 0.456 & $<0.001$ & 0.481 \\
\hline KIRP & Kidney renal papillary cell carcinoma & 0.511 & \multicolumn{2}{|c|}{ No or weak correlation } \\
\hline LGG & Brain Lower Grade Glioma & 0.558 & $<0.001$ & 0.462 \\
\hline STAD & Stomach adenocarcinoma & 0.588 & 0.002 & 0.455 \\
\hline LIHC & Liver hepatocellular carcinoma & 0.805 & \multicolumn{2}{|c|}{ No or weak correlation } \\
\hline BRCA & Breast invasive carcinoma & 0.808 & \multicolumn{2}{|c|}{ No or weak correlation } \\
\hline CESC & $\begin{array}{l}\text { Cervical squamous cell carcinoma and } \\
\text { endocervical adenocarcinoma }\end{array}$ & 0.982 & $<0.001$ & 0.412 \\
\hline COAD & Colon adenocarcinoma & \multirow{5}{*}{ Value not shown } & \multirow{5}{*}{\multicolumn{2}{|c|}{ No or weak correlation }} \\
\hline $\mathrm{KICH}$ & Kidney chromophobe & & & \\
\hline READ & Rectum adenocarcinoma & & & \\
\hline SKCM & Skin cutaneous melanoma & & & \\
\hline UCEC & Uterine corpus endometrioid carcinoma & & & \\
\hline
\end{tabular}

a

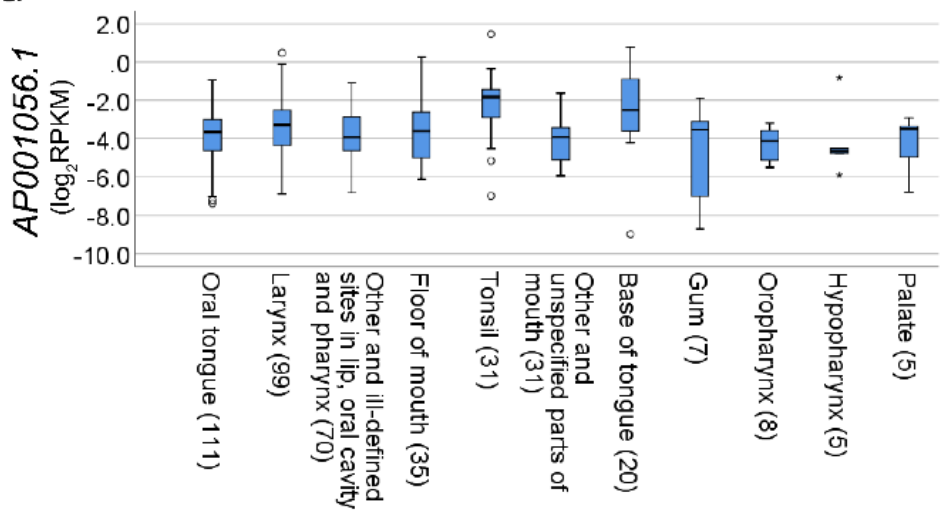

b

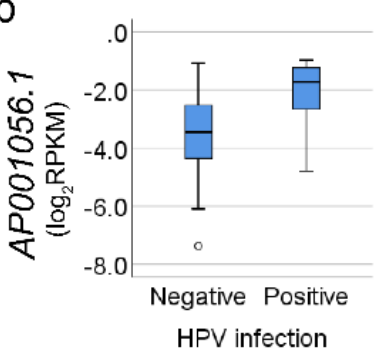

C

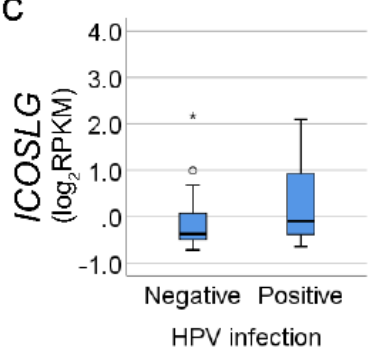

Figure 2. Gene expression related to tumor subsite and human papillomavirus (HPV) status. (a) Boxplots showing levels of AP001056.1 in different tumor subsites. Values in brackets show number of patients. $(\mathbf{b}, \mathbf{c})$ Boxplots showing levels of AP001056.1 and ICOSLG in HPV-positive and HPV-negative SCCHN tumors. Small circles indicate outliers and asterisks, $\left({ }^{*}\right)$ extreme outliers. 
TCGA data were available for HPV in situ hybridization from 89 SCCHN patients (67 HPV-negative and 22 HPV-positive). Of these, AP001056.1 and ICOSLG mRNA levels were available for $12 \mathrm{HPV}$-positive and $60 \mathrm{HPV}$-negative patients. The majority of HPV-positive tumors were from the tonsil (9 out of 12). The HPV-positive tumors showed higher levels of AP001056.1 compared to HPV-negative tumors ( $p=0.001$, Figure $2 b$ ). Similarly, ICOSLG mRNA levels were also higher in HPV-positive tumors ( $p=0.028$, Figure 2c). To further evaluate the function of AP001056.1, we used the TANRIC tool to identify significantly co-expressed genes in SCCHN. A total of 1641 transcripts showed a significant correlation with AP001056.1 $(p<0.001)$, including ICOSLG. Gorilla functional enrichment analysis revealed that the most significantly enriched biological process was a so-called "immune system process" (adjusted $p<0.001$ ). A list of the immune genes including Spearman correlation coefficients $\geq 0.6$ are shown in Table 3 .

Table 3. List of immune genes associated with AP001056.1 expression $(r \geq 0.600, p<0.001)$.

\begin{tabular}{|c|c|c|c|c|c|}
\hline Gene Symbol & $\begin{array}{l}\text { Spearman } \\
\text { Correlation } \\
\text { Coefficient } r\end{array}$ & Gene Symbol & $\begin{array}{l}\text { Spearman } \\
\text { Correlation } \\
\text { Coefficient } r\end{array}$ & Gene Symbol & $\begin{array}{l}\text { Spearman } \\
\text { Correlation } \\
\text { Coefficient } r\end{array}$ \\
\hline LRMP & 0.702 & IRF8 & 0.646 & ENPP2 & 0.627 \\
\hline NLRC3 & 0.696 & ITK & 0.645 & $\mathrm{RHOH}$ & 0.625 \\
\hline DOCK2 & 0.691 & NFAM1 & 0.644 & FCGR2C & 0.625 \\
\hline PIK3CG & 0.687 & ATP8B4 & 0.644 & CR1 & 0.625 \\
\hline PTPRC & 0.683 & FGR & 0.643 & LCP2 & 0.623 \\
\hline CD180 & 0.683 & ALOX5 & 0.643 & TLR10 & 0.618 \\
\hline BTK & 0.683 & NCF4 & 0.641 & FLT3 & 0.618 \\
\hline ARHGAP9 & 0.683 & CYSLTR1 & 0.641 & CTSS & 0.617 \\
\hline IKZF1 & 0.677 & SPN & 0.640 & SELL & 0.614 \\
\hline IL16 & 0.674 & HVCN1 & 0.639 & CD48 & 0.614 \\
\hline BIN2 & 0.670 & CCR2 & 0.639 & SELPLG & 0.613 \\
\hline APBB1IP & 0.670 & BTLA & 0.639 & LY9 & 0.613 \\
\hline SLAMF1 & 0.668 & TLR7 & 0.638 & CXCR4 & 0.613 \\
\hline NCKAP1L & 0.666 & CD5 & 0.637 & CORO1A & 0.613 \\
\hline CD53 & 0.664 & PRKCB & 0.636 & CD96 & 0.613 \\
\hline TLR1 & 0.663 & PREX1 & 0.635 & ATP8A1 & 0.612 \\
\hline PLCL2 & 0.662 & CD79B & 0.635 & ITGAX & 0.611 \\
\hline JAK3 & 0.659 & TNFRSF1B & 0.634 & CYBB & 0.611 \\
\hline TNFSF8 & 0.658 & DOCK8 & 0.634 & $\mathrm{CD} 27$ & 0.610 \\
\hline VAV1 & 0.657 & SLAMF6 & 0.633 & GAPT & 0.609 \\
\hline IKZF3 & 0.656 & ITGAM & 0.633 & CD4 & 0.609 \\
\hline NAIP & 0.655 & GPR174 & 0.633 & GPR65 & 0.608 \\
\hline DOK3 & 0.655 & CD84 & 0.633 & P2RX1 & 0.606 \\
\hline LILRB1 & 0.654 & PRAM1 & 0.632 & DOCK10 & 0.606 \\
\hline ZAP70 & 0.652 & IL21R & 0.632 & IRF4 & 0.603 \\
\hline WAS & 0.651 & BCL2 & 0.631 & CIITA & 0.603 \\
\hline TBC1D10C & 0.649 & LAT2 & 0.630 & IL2RG & 0.602 \\
\hline LTA & 0.649 & CCR4 & 0.628 & AMICA1 & 0.602 \\
\hline CECR1 & 0.648 & LILRA1 & 0.627 & CD3G & 0.600 \\
\hline CD28 & 0.648 & LAX1 & 0.627 & & \\
\hline ITGAL & 0.646 & LAT & 0.627 & & \\
\hline
\end{tabular}

\subsection{Validation of AP001056.1 and ICOSLG Levels by Reverse Transcription Quantitative PCR (RT-qPCR)}

RT-qPCR was performed to investigate gene expression and the correlation between AP001056.1 and ICOSLG in matched tumor-free and tumor samples from 12 patients with squamous cell carcinoma of the oral tongue (SCCOT), the most common head and neck cancer subtype [19]. Up-regulation of AP001056.1 and down-regulation of ICOSLG was seen in tumors compared to tumor-free samples $(p=0.002$, Figure $3 \mathrm{a}, \mathrm{b})$, and a significantly positive correlation between these two RNAs was seen 
in the tumors (Spearman's rank correlation coefficient $r=0.867, p<0.001$ ) but not in the tumor-free controls (Figure 3c).

a

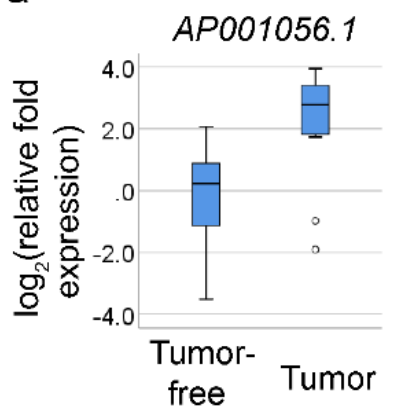

b

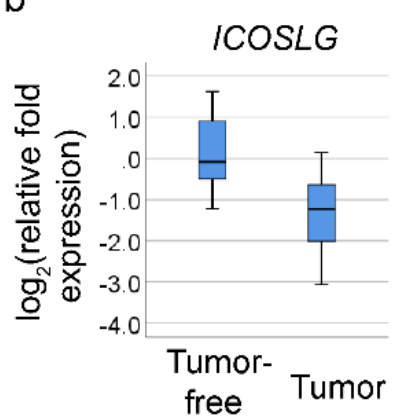

C

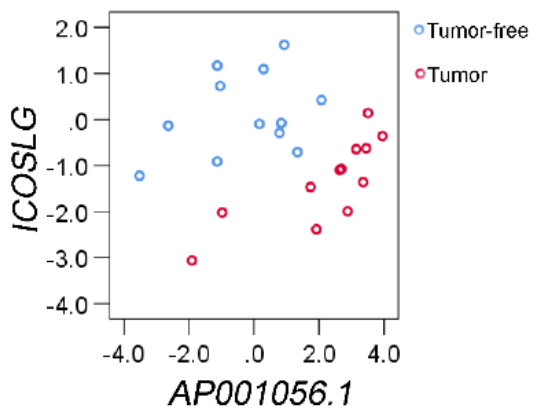

Figure 3. Quantification of AP001056.1 and ICOSLG levels by RT-qPCR. (a,b) Boxplots showing log transformed relative fold levels of AP001056.1 and ICOSLG in matched tumor-free and tumor samples from 12 patients with oral tongue SCC. Small circles indicate outliers. (c) Scatterplot showing correlation between AP001056.1 and ICOSLG mRNA in tumor-free and tumor samples.

\section{Discussion}

eRNAs are a specific subclass of lncRNAs derived within gene enhancer regions and are able to act in cis to influence transcription of the corresponding gene. To improve our understanding of eRNAs in SCCHN, we set out to identify prognosis-related eRNAs in SCCHN using the following approach, we: (1) Searched for lncRNAs expressed from active tissue-specific enhancers as putative eRNAs, (2) identified a subset of these putative eRNAs that significantly correlated with overall survival and (3) from these, identified a further subset that correlated with their potential target. This novel approach enriches for putative eRNAs influencing SCCHN pathobiology and can be employed for other human diseases with sufficient RNA-sequencing data available.

According to our criteria, the functionally unannotated lncRNA AP001056.1 was identified as the top key eRNA candidate in SCCHN. AP001056.1 is located within the enhancer region of the ICOSLG gene. Its encoded protein, ICOSLG, is a ligand for the T-cell-specific cell surface receptor ICOS and acts as a costimulatory signal for T-cell proliferation and cytokine secretion [20]. Strong correlations between expression of AP001056.1 and ICOSLG were seen in several types of cancer, whereas, the most significant impact on overall survival was seen in SCCHN, making it an interesting lncRNA for this tumor type.

A recent study showed evidence for an association between HPV and expression of ICOSLG in SCCHN [21]. We found AP001056.1 to be present at higher levels in HPV-positive compared to HPV-negative SCCHN, and when comparing AP001056.1 levels between tumors from different subsites, the highest level was seen in tumors from the tonsil, probably due to the fact that the majority of tonsillar cancer are HPV-positive [22]. These results once again emphasize the necessity to take the subsite into consideration when interpreting data from analysis of the group of SCCHN tumors.

As AP001056.1 is a functionally unannotated transcript, we tried to clarify its role by identifying other co-expressed genes in addition to its predicted target ICOSLG. Surprisingly, besides ICOSLG, we found a total of 1640 genes with significant expression correlations with AP001056.1. Although eRNA functions are primarily performed in cis, several observations suggest that eRNA could mediate the expression of other genes in trans [7,12]. AP001056.1 could thus be speculated to have direct or indirect trans effects. Still, correlations between transcripts do not necessarily imply causal relationships, and whether AP001056.1 is a functional component of enhancer activity remains to be determined. Nevertheless, according to gene ontology enrichment analysis, the gene transcripts that correlated to AP001056.1 are mainly involved in immune system processes. Together with the correlation with the immune checkpoint protein ICOSLG, these results indicate that AP001056.1 has a 
role in regulating the immune response in SCCHN. Considering the disease-specific impact on patient survival, novel therapeutic approaches that could increase AP001056.1 expression might help to induce protective immunity for effective treatment of these cancers.

\section{Materials and Methods}

\subsection{Identifying Prognostic eRNAs in SCCHN through Integrated Data Analysis}

A list of lncRNAs expressed from active tissue-specific enhancers and their assigned targets predicted by PreSTIGE $[18,23]$ was obtained. Ensembl BioMart was used to obtain the mapping between Ensembl transcript ID and gene symbol. The levels of putative eRNAs and their clinical relevance in the SCCHN cohort in TCGA were investigated using the interactive web server TANRIC (the Atlas of Noncoding RNAs in Cancer) [24]. The TANRIC co-expression data were also studied to evaluate correlations between putative eRNA levels and their predicted target genes. Putative eRNAs with significant correlations with both overall survival $(p<0.05)$ and levels of their target genes $(p<0.001)$ were considered candidate key eRNAs in SCCHN. Next, the TANRIC IncRNA and corresponding target gene expression levels were studied across 20 cancer types to evaluate the differences related to cancer type. Finally, we downloaded TCGA SCCHN RNA-sequencing and clinical data using cBioPortal [25] to investigate differences in gene expression related to SCCHN tumor subsite and HPV status.

\subsection{Gene Ontology Enrichment Analysis}

Besides the predicted targets, other transcripts with significant correlation with the identified prognostic eRNAs were obtained through TANRIC. To investigate the possible functional characteristics of the eRNA related coding genes, gene ontology enrichment analysis was performed (identification of gene ontology terms that are significantly overrepresented in a given set of genes [26]) using the web-based tool Gorilla [27]. The enriched gene ontology terms with an adjusted $p$-value $<0.001$ were considered as relevant biological processes.

\subsection{Quantification of AP001056.1 and ICOSLG Transcript Levels in Tongue Cancer Samples}

Reverse transcription quantitative PCR (RT-qPCR) was used to confirm the RNA-sequencing data. The transcript ID is ENST00000411956.1 (for gene AP001056.1) and ENST00000407780.7 (for gene ICOSLG). The isolated total RNA was sourced from matched tumor and tumor-free samples from twelve patients diagnosed with squamous cell carcinoma of the oral tongue (SCCOT) [28,29]. The study was approved by the Regional Ethics Review Board, Umeå, Sweden (Dnr 03-201 and Dnr 08-003 $\mathrm{M})$ and performed in accordance with the Declaration of Helsinki. The written informed consent was obtained from all patients and healthy individuals. The cDNA was synthesized from $500 \mathrm{ng}$ total RNA using the RevertAid H minus first strand cDNA synthesis kit (Thermo Fisher Scientific, Waltham, MA, USA). RT-qPCR was performed using an IQ5 multicolor real-time PCR detection system with IQ SYBR Green Supermix (Bio-Rad Laboratories, Hercules, CA, USA). Custom primers for AP001056.1 (forward: ATGGCCAGGCTGATTTCGAA, reverse: TCCAGATGAAAATGCCGGCT) and ICOSLG (forward: TCTGCAGCAGAACCTGACTG, reverse: TTTCTCGCCGGTACTGACTG) were obtained from Thermo Fisher Scientific. Each sample was measured in triplicate and the results were normalized to the housekeeping gene RPL13A (forward: GTACGCTGTGAAGGCATCAA, reverse: GTTGGTGTTCATCCGCTTG) and GAPDH (sequences of primers not provided, Primerdesign Ltd, Southampton, United Kingdom).

\subsection{Statistics}

To compare gene expression among different tumor subsites, the non-parametric Kruskal-Wallis $\mathrm{H}$ test was performed and the non-parametric Wilcoxon signed-rank test was used to compare between matched tumor and tumor-free samples. Spearman's rank correlation coefficient was calculated to 
evaluate correlation strength. All statistical tests were conducted in IBM SPSS Statistics 25. A two-sided $p$-value $<0.05$ was considered statistically significant.

\section{Conclusions}

To conclude, we developed and applied a novel approach to identify key eRNAs in SCCHN. Our data suggest that lncRNA AP001056.1 is a prognosis-related gene for SCCHN, functioning as a tissue-specific eRNA of ICOSLG. With a potential role in immune response, AP001056.1 is a promising therapeutic target for patients with this tumor type when taking subsite into consideration.

Author Contributions: X.G., L.W. and K.N. conceived and designed the study; X.G. and L.B. performed the experiments; X.G. and L.W. analyzed the data; T.W. contributed medical materials; X.G. and L.W. prepared the original draft; P.J.C., R.F., N.S. and K.N. made revisions; K.N. supervised the project; all authors approved the final version of the manuscript.

Funding: This study was supported by Lion's Cancer Research Foundation, Umeå University (K.N. and R.F.); The Swedish Cancer Society (contract number 180542 and 1802 96); Umeå University; Västerbottens Läns Landsting in Sweden; and project MEYS-NPS I-LO1413 and MH CZ-DRO (MMCI, 00209805) in the Czech Republic.

Conflicts of Interest: The authors declare no conflict of interest. The funders had no role in the design of the study; in the collection, analyses, or interpretation of data; in the writing of the manuscript, or in the decision to publish the results.

\section{References}

1. Bray, F.; Ferlay, J.; Soerjomataram, I.; Siegel, R.L.; Torre, L.A.; Jemal, A. Global cancer statistics 2018: GLOBOCAN estimates of incidence and mortality worldwide for 36 cancers in 185 countries. CA Cancer J. Clin. 2018. [CrossRef] [PubMed]

2. Spence, T.; Bruce, J.; Yip, K.W.; Liu, F.F. HPV associated head and neck cancer. Cancers 2016, 8, 75. [CrossRef] [PubMed]

3. Leemans, C.R.; Snijders, P.J.F.; Brakenhoff, R.H. The molecular landscape of head and neck cancer. Nat. Rev. Cancer 2018, 18, 269-282. [CrossRef] [PubMed]

4. Arun, G.; Diermeier, S.D.; Spector, D.L. Therapeutic targeting of long non-coding RNAs in cancer. Trends Mol. Med. 2018, 24, 257-277. [CrossRef] [PubMed]

5. Atianand, M.K.; Caffrey, D.R.; Fitzgerald, K.A. Immunobiology of long noncoding RNAs. Annu. Rev. Immunol. 2017, 35, 177-198. [CrossRef] [PubMed]

6. Kopp, F.; Mendell, J.T. Functional classification and experimental dissection of long noncoding RNAs. Cell 2018, 172, 393-407. [CrossRef] [PubMed]

7. Ransohoff, J.D.; Wei, Y.; Khavari, P.A. The functions and unique features of long intergenic non-coding RNA. Nat. Rev. Mol. Cell. Biol. 2018, 19, 143-157. [CrossRef] [PubMed]

8. Iyer, M.K.; Niknafs, Y.S.; Malik, R.; Singhal, U.; Sahu, A.; Hosono, Y.; Barrette, T.R.; Prensner, J.R.; Evans, J.R.; Zhao, S.; et al. The landscape of long noncoding RNAs in the human transcriptome. Nat. Genet. 2015, 47, 199-208. [CrossRef] [PubMed]

9. Song, W.; Sun, Y.; Lin, J.; Bi, X. Current research on head and neck cancer-associated long noncoding RNAs. Oncotarget 2018, 9, 1403-1425. [CrossRef] [PubMed]

10. Nohata, N.; Abba, M.C.; Gutkind, J.S. Unraveling the oral cancer lncRNAome: Identification of novel lncRNAs associated with malignant progression and HPV infection. Oral Oncol. 2016, 59, 58-66. [CrossRef] [PubMed]

11. Natoli, G.; Andrau, J.C. Noncoding transcription at enhancers: General principles and functional models. Annu. Rev. Genet 2012, 46, 1-19. [CrossRef] [PubMed]

12. Chen, H.J.; Du, G.S.; Song, X.; Li, L. Non-coding transcripts from enhancers: New insights into enhancer activity and gene expression regulation. Genom. Proteom. Bioinform. 2017, 15, 201-207. [CrossRef] [PubMed]

13. Fang, Y.; Fullwood, M.J. Roles, functions, and mechanisms of long non-coding RNAs in Cancer. Genom. Proteom. Bioinform. 2016, 14, 42-54. [CrossRef] [PubMed]

14. Liu, Y.; Ding, M.; Gao, Q.; He, A.; Liu, Y.; Mei, H. Current advances on the important roles of enhancer RNAs in gene regulation and cancer. Biomed. Res. Int. 2018, 2018, 2405351. [CrossRef] [PubMed] 
15. Bal, E.; Park, H.S.; Belaid-Choucair, Z.; Kayserili, H.; Naville, M.; Madrange, M.; Chiticariu, E.; Hadj-Rabia, S.; Cagnard, N.; Kuonen, F.; et al. Mutations in ACTRT1 and its enhancer RNA elements lead to aberrant activation of Hedgehog signaling in inherited and sporadic basal cell carcinomas. Nat. Med. 2017, 23, 1226-1233. [CrossRef] [PubMed]

16. Irimie, A.I.; Zimta, A.A.; Ciocan, C.; Mehterov, N.; Dudea, D.; Braicu, C.; Berindan-Neagoe, I. The unforeseen non-coding RNAs in head and neck cancer. Genes 2018, 9, 134. [CrossRef] [PubMed]

17. Consortium, E.P. An integrated encyclopedia of DNA elements in the human genome. Nature 2012, 489, 57-74. [CrossRef] [PubMed]

18. Vucicevic, D.; Corradin, O.; Ntini, E.; Scacheri, P.C.; Orom, U.A. Long ncRNA expression associates with tissue-specific enhancers. Cell Cycle 2015, 14, 253-260. [CrossRef] [PubMed]

19. Mascitti, M.; Rubini, C.; De Michele, F.; Balercia, P.; Girotto, R.; Troiano, G.; Lo Muzio, L.; Santarelli, A. American Joint Committee on Cancer staging system 7th edition versus 8th edition: Any improvement for patients with squamous cell carcinoma of the tongue? Oral. Surg. Oral. Med. Oral. Pathol. Oral. Radiol. 2018, 126, 415-423. [CrossRef] [PubMed]

20. Nurieva, R.I.; Mai, X.M.; Forbush, K.; Bevan, M.J.; Dong, C. B7h is required for T cell activation, differentiation, and effector function. Proc. Natl. Acad. Sci. USA 2003, 100, 14163-14168. [CrossRef] [PubMed]

21. Wood, O.; Woo, J.; Seumois, G.; Savelyeva, N.; McCann, K.J.; Singh, D.; Jones, T.; Peel, L.; Breen, M.S.; Ward, M.; et al. Gene expression analysis of TIL rich HPV-driven head and neck tumors reveals a distinct B-cell signature when compared to HPV independent tumors. Oncotarget 2016, 7, 56781-56797. [CrossRef] [PubMed]

22. Loizou, C.; Laurell, G.; Lindquist, D.; Öfverman, C.; Stefansson, K.; Nylander, K.; Olofsson, K. Incidence of tonsillar cancer in northern Sweden: Impact of human papilloma virus. Oncol. Lett. 2015. [CrossRef] [PubMed]

23. Corradin, O.; Saiakhova, A.; Akhtar-Zaidi, B.; Myeroff, L.; Willis, J.; Cowper-Sal lari, R.; Lupien, M.; Markowitz, S.; Scacheri, P.C. Combinatorial effects of multiple enhancer variants in linkage disequilibrium dictate levels of gene expression to confer susceptibility to common traits. Genome Res. 2014, 24, 1-13. [CrossRef] [PubMed]

24. Li, J.; Han, L.; Roebuck, P.; Diao, L.; Liu, L.; Yuan, Y.; Weinstein, J.N.; Liang, H. TANRIC: An Interactive Open Platform to Explore the Function of lncRNAs in Cancer. Cancer Res. 2015, 75, 3728-3737. [CrossRef] [PubMed]

25. Gao, J.; Aksoy, B.A.; Dogrusoz, U.; Dresdner, G.; Gross, B.; Sumer, S.O.; Sun, Y.; Jacobsen, A.; Sinha, R.; Larsson, E.; et al. Integrative analysis of complex cancer genomics and clinical profiles using the cBioPortal. Sci. Signal 2013, 6, p11. [CrossRef] [PubMed]

26. Khatri, P.; Draghici, S. Ontological analysis of gene expression data: Current tools, limitations, and open problems. Bioinformatics 2005, 21, 3587-3595. [CrossRef] [PubMed]

27. Eden, E.; Navon, R.; Steinfeld, I.; Lipson, D.; Yakhini, Z. GOrilla: A tool for discovery and visualization of enriched GO terms in ranked gene lists. BMC Bioinform. 2009, 10, 48. [CrossRef] [PubMed]

28. Boldrup, L.; Gu, X.; Coates, P.J.; Norberg-Spaak, L.; Fahraeus, R.; Laurell, G.; Wilms, T.; Nylander, K. Gene expression changes in tumor free tongue tissue adjacent to tongue squamous cell carcinoma. Oncotarget 2017, 8, 19389-19402. [CrossRef] [PubMed]

29. Gu, X.; Boldrup, L.; Coates, P.J.; Fahraeus, R.; Nylander, E.; Loizou, C.; Olofsson, K.; Norberg-Spaak, L.; Garskog, O.; Nylander, K. Epigenetic regulation of OAS2 shows disease-specific DNA methylation profiles at individual CpG sites. Sci. Rep. 2016, 6, 32579. [CrossRef] [PubMed]

(c) 2019 by the authors. Licensee MDPI, Basel, Switzerland. This article is an open access article distributed under the terms and conditions of the Creative Commons Attribution (CC BY) license (http://creativecommons.org/licenses/by/4.0/). 